\title{
Design of a Current Sensing Circuit without Power Loss for DC-DC Converter
}

\author{
Liu Shulin \\ Xi'an University of Science \& Technology \\ Xi'an, China \\ 1sigma@163.com
}

\author{
Qi Lili \\ Xi'an University of Science \& Technology \\ Xi'an, China \\ qiyubianli@163.com
}

\begin{abstract}
In order to decrease the additional power loss in DC-DC converter caused by current detection, a current sensing technique without power loss is proposed. Power MOSFET is being widely used in low and medium power DC-DC converters; it can be regarded as a resistor in the condition of turn-on. Thus, based on the analysis of traditional kinds of current detection technology, a novel current sensing technology is proposed, which senses the current by using the conduction voltage drop of the power devices in DC-DC converter, the accuracy of sensing current can be improved by adjusting the resistor connected in both ends of the transconductance amplifier, no additional current sensing circuit is needed, and the additional power loss can be avoided. The DC-DC converter operating at the frequency of $1 \mathrm{MHz}$ is simulated, and the simulation results verify the accuracy and feasibility of the designed circuit.
\end{abstract}

Keywords-DC-DC converter;current mode;over-current protection;conduction voltage drop;current sensing technology without power loss

\section{INTRODUCTION .}

As energy crisis exist in the modern society, under the premise of the appropriate power conversion indicators and performance, saving energy and reducing energy consuming maximally is still the goal for chip switching power supply integrated circuits. Because of its high conversion efficiency, broadly input dynamic range and small size, light weight, etc., the switching power supply will be always used in the current sensing circuits $[1,2]$. With the development of economic technology, the operating voltage of apparatus and chip is more and more low, thus, the step-down converters are being widely in the industry $[3,4]$.

In the current mode control, how to detect the current through the switch tube accurately to realize the control of current loop is a big problem. Because the accuracy of current detection and the power consumption is contradictory, more accuracy means more power consumption. In the power system, the current detection is more than one point; the current detection signal is being widely used in the following aspects [5]:

(1)Controlling current loop, the detected peek current and given current are compared to adjust duty cycle to achieve stable output voltage.

(2)Limiting the maximum current to achieve overcurrent protection, it is often used with (1) together.

(3)Detecting current in every phrase to achieve current sharing in the poly-phase system.
(4)Detecting the average output current to achieve the lower output voltage with load current in the active lowered voltage system.

The current sensing technology being widely used is to detect current by using the characteristic that the parallel MOSFET is proportional. The breadth length ratio between SENSEFET and MOSFET is $1 / \mathrm{n}$, the current through SENSEFET decreases to $1 / n$ current through the power transistor. A resistor existed in the end of the SENSEFET, a basic current sensing circuit is designed, the current through SENSEFET can be detected by detecting the voltage in the resistor, and then the current through MOSFET can be obtained. However, the power loss caused by the resistor is large; it is difficult to be controlled [6].

Another way to detect the conduction voltage is to regard the switch device to a resistor, no extra power consumption is not exist, so it is a current sensing technology without power loss. However, the resistance changes with the change of temperature, and the detection accuracy is not high [7]. Thus, novel current sensing technology has become the research orientation for the switch power supply [8,9], and the design of current sensing has also become a focused issue in the industry $[10,11]$.

The current can be sensed by using the conduction voltage drop of the power devices in DC-DC converter in this paper, no additional power loss is existed. The transconductance amplifier and the dynatron are used to form a negative feedback loop, and the output current is mirrored exported with current mirror, the accuracy of current detection can be improved by adjusting the resistor connected in both ends of the transconductance amplifier and current sensing without power loss can be achieved.

\section{TRADITIONAL KINDS OF CURRENT DETECTION TECHNOLOGY}

The resistor is in series with the inductor in traditional current sampling circuit, as shown in Fig .1. The current through inductor can be obtained according to the volatge in the resistor in the condition of the given resistance.

However, the current through inductor and the leakage current of the power device must flow through the sampling resistor, the power loss existed in the sampling resistor will lead to the lower efficiency of DC-DC converters. Supposing the current is $1 \mathrm{~A}$ in the full load, the voltage drop in the sampling resistor is $100 \mathrm{mV}$, and the power loss of the resistor is $0.1 \mathrm{~W}$; if the required output power is $3.3 \mathrm{~W}$, the efficiency will decrease $3.3 \%$ in the 
condition of using sampling resistor. If the output voltage is lower, the power loss of the resistor is more serious, and the efficiency is lower. The shortcomings will be more serious in the applications with lower voltage and high current.

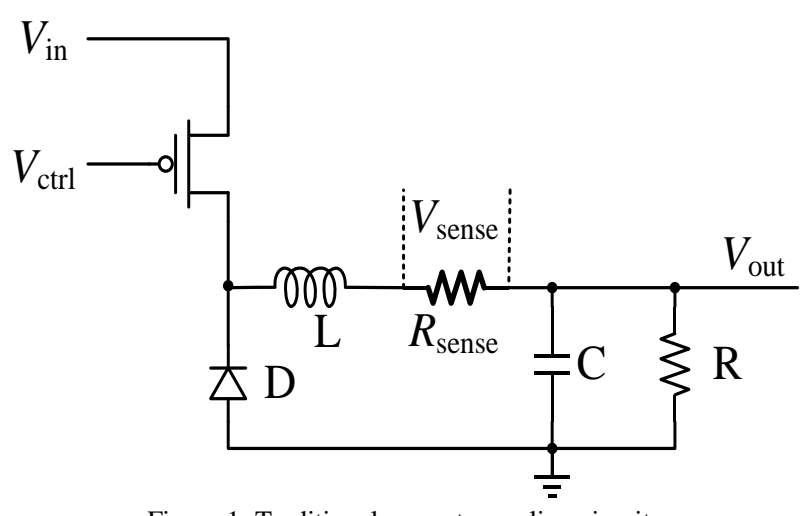

Figure 1. Traditional current sampling circuit

SENSE FETs sampling technology is being widely used in novel power MOSFET circuits, its principle is shown in Fig .2. The grid and the source of power transistor are connected to the sample transistor respectively, if the drain voltage in two transistors are equal, the power transistor is in parallel with the sample transistor, and the current in sample transistor corresponds to the scaled-down current in power transistor. The effective channel width of the sample transistor should be very small to ensure its power loss be very low, and it can be regarded as lossless. The voltage in $\mathrm{M}$ and $\mathrm{S}$ should be equal to eliminate the current mismatch caused by channel length modulation.

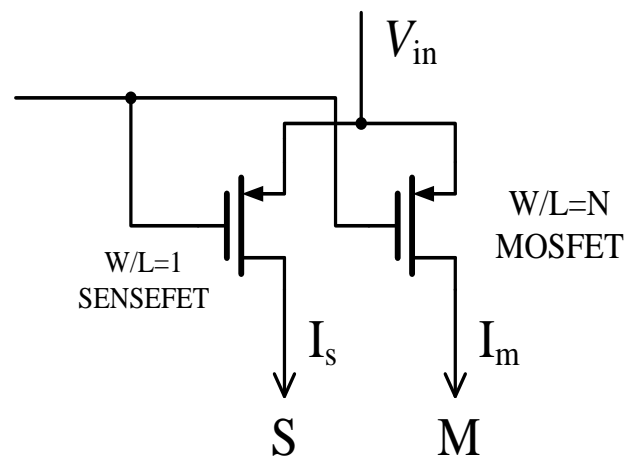

Figure 2. SENSE FETs sampling circuit

The entire SENSE FETs sampling circuit is shown in Fig .3. Where, M1 is the power MOS transistor, M2 is the sampling transistor, the operational amplifier is used to ensure the drain voltages in two transistor be equal. The accuracy relys on the matching performance of the current mirror.

With the increase of the channel width ratio between the power MOS transistor and the sampling transistor, the matching performance will decrease, and the accuracy in the entire system will also decrease, and the error can reach to $120 \%$. Considering the current ratio is 100 : 1 , a small couple between the power MOS transistor and the sampling transistor will cause a large error, and a large peak signal will be added to the sampling signal. Thus, the IC layout must be designed carefully to insure that the mutual inductance between the power MOS transistor and the sampling transistor is the smallest. Besides, the technology can only be used when the MOSFET is matched highly.

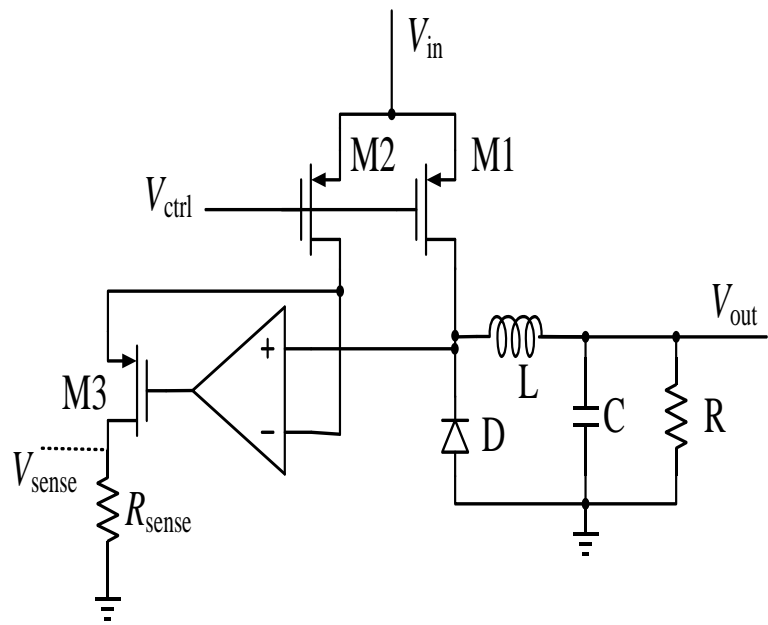

Figure 3. Entire SENSE FETs sampling circuit

Another current sampling technology is shown in Fig .4. The on-resistor RDS in MOSFET power transistor is used to sample current. If MOS power transistor operates in linear region, it can be regarded as a switch, and it can be seen as an equivalent resistor RDS, RDS can be expressed by:

$$
R_{\mathrm{DS}}=\frac{L}{W \mu C_{o x}\left(V_{G S}-V_{T}\right)}
$$

Where, $\mu$ is the channel charge mobility, Cox is the unit gate oxide capacitance, VT is the threshold voltage. W is the effective width of the power MOSFET, L is the effective length of the conducting channel.

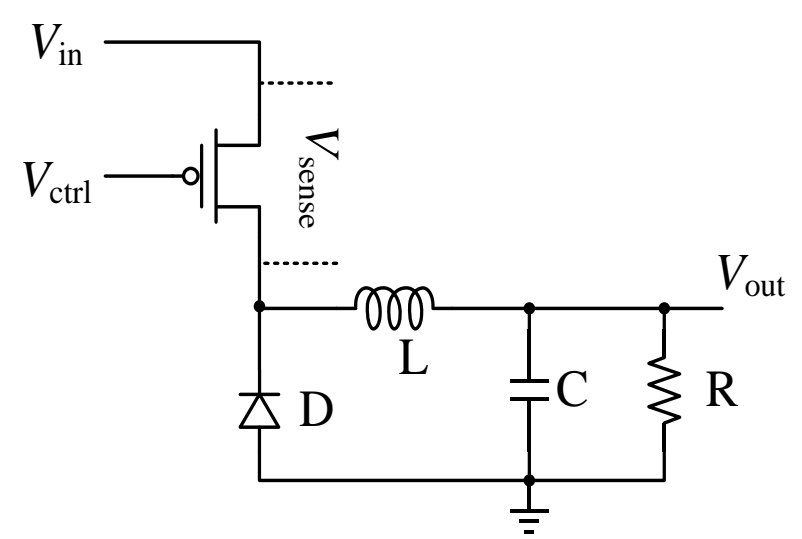

Figure 4.Sampling with the power MOSFET resistor

Thus, the current can be obtained if the RDS is given and the voltage between the source and the drain can be measured. RDS should be controlled well in this technology. If RDS is too small, the small sampling signal can not drive the lower circuit, the signal should be enlarge by amplifier. If RDS is too large, the efficiency in converters will be reduced by its power loss, especially in low voltage and large current situation.

Besides, RDS is nonlinear inherently because of the process factor, Cox and VT will change with the change of temperature, thus, a nonlinear change existed in the RDS 
of the MOSFET, and the largest error can reach from $50 \%$ to $100 \%$, so the accuracy in this technology is limited.

However, there is no extra resistor in the sampling circuit, the extra power loss is not existed, so the efficiency is higher and the technology is being widely used.

The current is sensed by detecting the voltage in synchronous rectifier in this technology.

\section{PRINCIPLE OF CURRENT SENSING CIRCUIT WITHOUT POWER LOSS}

A current sensing circuit without power loss using the voltage drop on the power device in DC-DC converter to detect the current is designed in this paper, the designed circuit has no additional power loss in relative to the entire system. The schematic is shown in Fig .5.

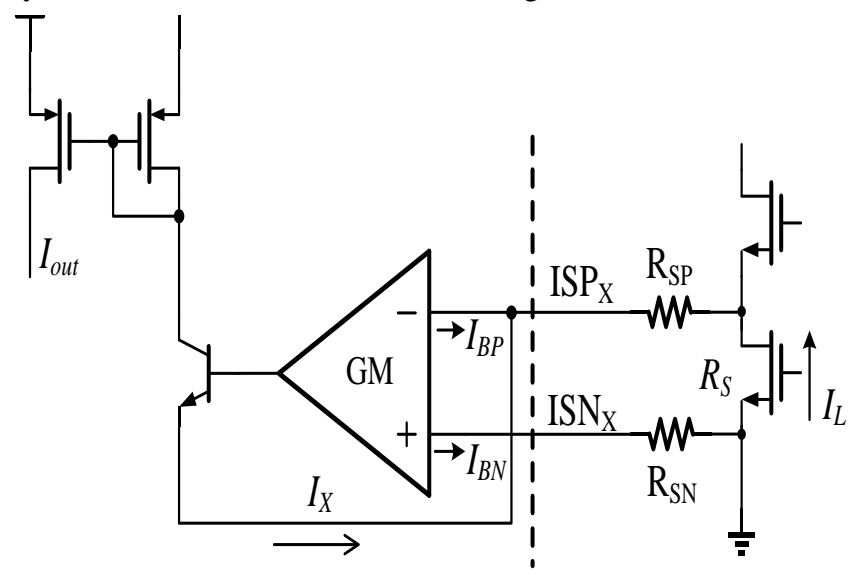

Figure 5. Current detection schematic

Negative feedback loop is used to ensure VISPX=VISNX. The sample current is mirrored exported with current mirror to achieve the following functions: Firstly, two-way currents are summed and then averaged, the averaged current is used as the benchmark, and the current difference of each phase is used to adjust the width of PWM. Secondly, the current is sent to over-current protection circuit to achieve the function of over-current protection. Finally, the current is sent to ripple regulator to adjust output voltage ripple. The current can be sensed by using the conduction voltage drop of the power devices in DC-DC converter in this paper, no additional power loss is existed. According to the above principle, we have

$$
I_{B P} \times R_{S P}+I_{X} \times R_{S P}-I_{L} \times R_{S}=I_{B N} \times R_{S N}
$$

As $I_{\mathrm{BP}}=I_{\mathrm{BN}}$, if $R_{\mathrm{SP}}=R_{\mathrm{SN}}$, the detecting current can be expressed by:

$$
I_{X}=\frac{I_{L} \times R_{S}}{R_{S P}}
$$

From (3), it can be seen that the detection current is proportional to the inductor current, and the detection progress and amplitude can be adjusted by the resistor series connected in the ends of the detection amplifier. The specific current detection schematic is shown in Fig .6.

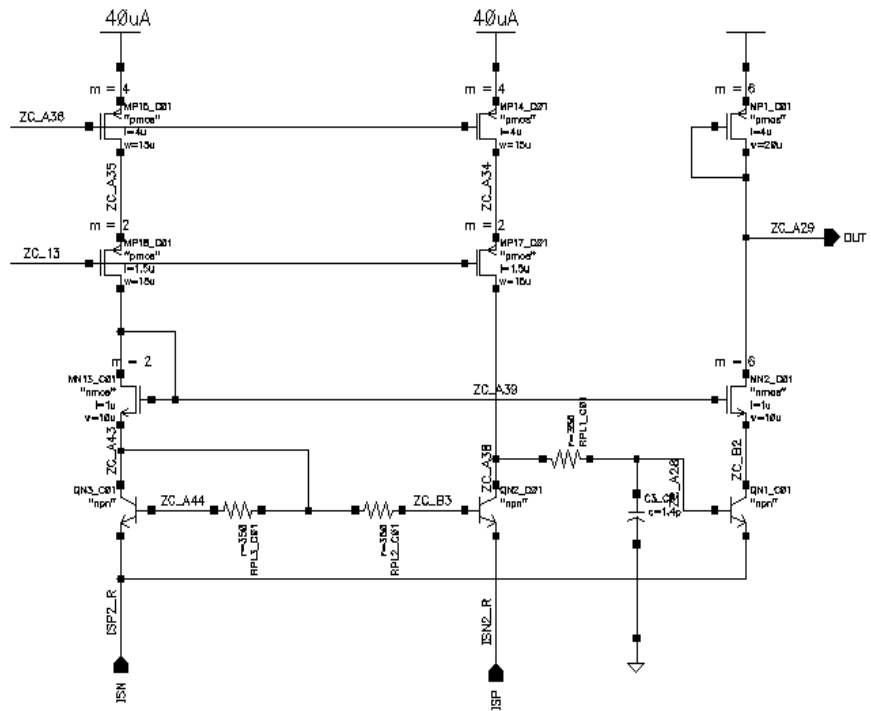

Figure 6. Specific current detection schematic

\section{SimUlATION}

Synchronous rectifier is replaced by a current source to simulate the output current. Its transient simulation waveforms are shown in Fig .7, the current through load Iout is simulated in the top picture, and the detected current Isense is simulated in the below picture, from Fig .8, it can be seen that the current change is successful detected in the condition of $1 \mathrm{MHz}$. The simulation results verify the accuracy and feasibility of the designed circuit.

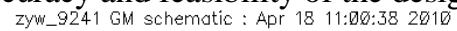
Transient Response
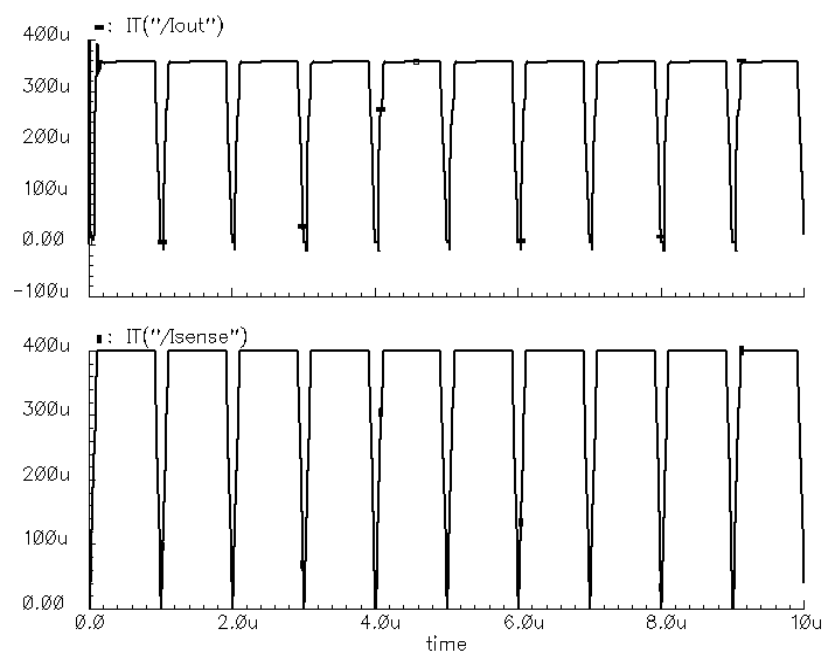

Figure 7. Current detection transient simulation waveforms

From equation (3), it can be seen that the current of the power device can not be tracked in the condition of the resistance of $\mathrm{RS}$ is too small (Supposing $\mathrm{RS}=0.5 \Omega$ ). The simulation results are shown in Fig .8. 


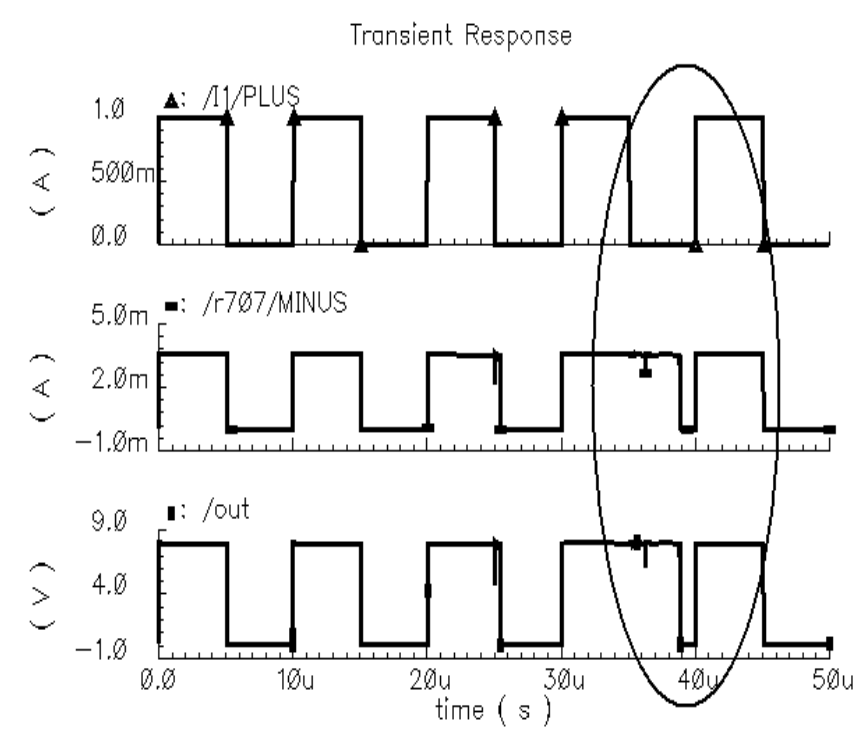

Figure 8. Failure to detect current change

The current track detection can be achieved by adjusting the resistor connected in the detection amplifier. The simulation results are shown in Fig .9.

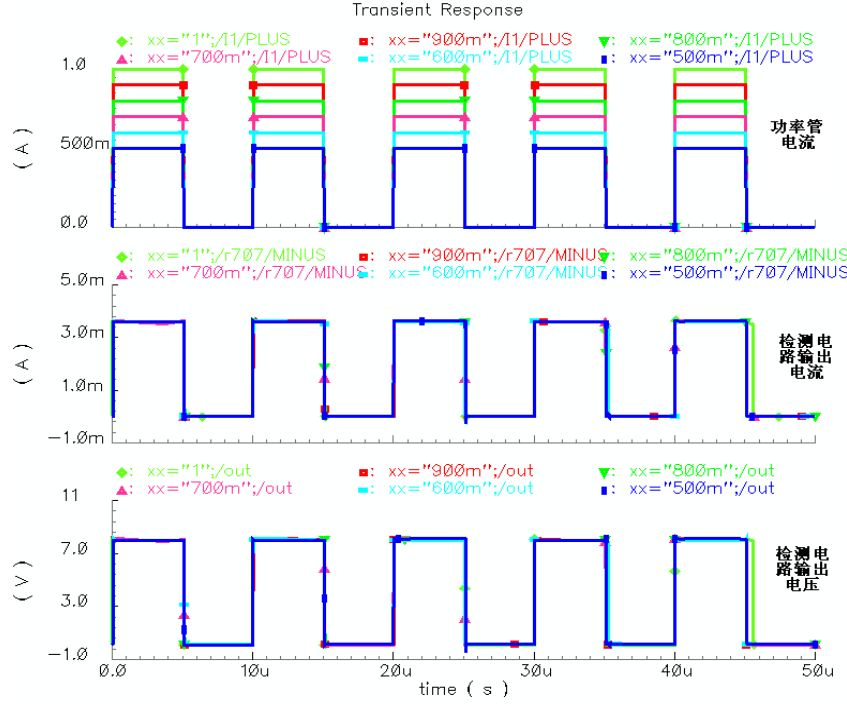

Figure 9. Success to detect current change

\section{CONCLUSION}

A novel current sensing technology without power loss is proposed in this paper, which detects the current using the conduction voltage drop of the power devices in DCDC converter, and the output current is mirrored exported with current mirror, the accuracy of current detection can be improved by adjusting the resistor connected in the transconductance amplifier, and current sensing without power loss can be achieved. The simulation results verify the feasibility of the designed circuit, and the low accuracy caused by the temperature can be eliminated. The technology has high availability and economic value, and it can be used in other current detection circuits.

\section{ACKNOWLEDGMENT}

This work is supported by the National Natural Science Foundation of China $(50977077,51277149)$.

\section{REFERENCES}

[1] Liu Shulin and Liu Jian. "Analyze and design of switching converters,"Beijing : Machinery Industry Press,2001.

[2] Zhang Zhansong and Cai Xuansan. "Principle and design of switching supply,'Beijing : Electronic IndustryPress, 1998.

[3] Liu Jixing, Shen jinfei. "A Novel Soft-switching BUCK Converter," Electrical Automation,vol.36(6),2014,pp.14-17.

[4] Ge Maoyan and Xie Lili. "Modeling and simulation of unideal buck converter," Computer Simulation, vol.27(4),2010,pp.333-336.

[5] Liu Bumin, Cai Wei ,Lu Tiejun and Wang Zongmin. "Low-Power Lossless Current Sensing in DC/DC Converter ," School of Microelectronics and Solid-State Electronics, University of Electronic Science and Technology of China Chengdu, vol.37(3), 2008,pp. 393-396

[6] Huang Zhigang, Zhang Bo and Tang Zhi. "Lossless Current Sensing in DC/DC Converter ," Electrotechnical Application, vol. 25(6), 2006, pp. 67-70.

[7] CHANG C H and CHANG R C. "A novel Current sensing Circuit for a current mod control CMOS DC-DC Buck converter," IEEE Int SymP Circuit and Systems, May.2005,pp. 120-123.

[8] Shi Liping and Liu Peng. "An Improved FBD Harmonic without PLL on and Reactive Currents Detection Method," Electrical Measurement\&Instrumentation, vol.16,2014,pp.56-61.

[9] Fang Yuhua and Zhao Menglian. "Reconfigurable High-efficiency DC-DC Converter with Lossless Current-sensing Technique," Journal of Zhejiang University, vol. 48(10),2014,pp. 1856-1864.

[10] Liu Wenxiao. "Design of Inductor Current Sensing Circuit for DCDC Converter ," Electronic Technology \& Software Engineering, vol. 19, 2013, pp. 113-113.

[11] Chi Shangsheng and Hu Wei. "A Modified Current Sensing Circuit for DC-DC Buck Converter,"Application of Integrated Circuits,vol.40(9),2014,pp.41-44. 